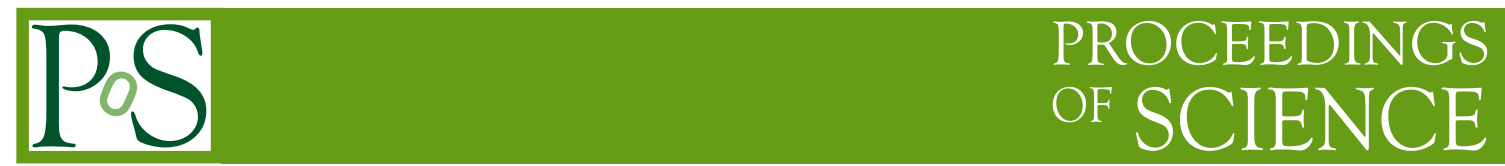

\title{
Searches for third generation squarks at the Tevatron
}

\section{Miguel VIDAL*ฑ}

Centro de Investigaciones Energéticas, Medioambientales y Tecnológicas (CIEMAT) -

Av. Complutense 22 Madrid, Spain.

E-mail: vidalefnal.gov

In the Minimal Supersymmetric Standard Model (MSSM), the large mixing between the chiral states of the scalar partner particles of the standard model fermions might lead to small masses for sbottom and stop quarks. Dedicated searches are carried out in final states with large missing transverse energy and at least two jets, where at least one of the jets is required to be b-tagged (sbottom searches) or c-tagged (stop searches). Most recent results from several analysis performed by the CDF and D0 collaborations are presented.

European Physical Society Europhysics Conference on High Energy Physics July 16-22, 2009

Krakow, Poland

${ }^{*}$ Speaker.

$\dagger$ On behalf of CDF and D0 Collaborations. 


\section{Introduction}

Supersymmetry (SUSY) [1] is regarded as one of the most compelling theories to describe physics at arbitrarily high energies beyond the Standard Model (SM). In SUSY, a new spin-based symmetry turns a bosonic state into a fermionic state - and vice versa - postulating the existence of a superpartner for each of the known fundamental particles, with spin differing by $1 / 2$ unit. The phenomenology is determined by the breaking mechanism of the symmetry and several constraints are assumed to reduce the vast SUSY parameter space. If R-parity [2] is conserved, SUSY particles have to be produced in pairs and ultimately decay into the lightest supersymmetric particle (LSP), usually identified as the lightest neutralino $\tilde{\chi}_{1}^{0}$, which constitutes a valid candidate for cold dark matter because it is colorless and neutral. Due to these properties the LSP escapes detection and it is identified as a large imbalance in transverse momentum historically called missing transverse energy $\left(\not_{T}\right)$.

\section{Third Generation Squarks Searches}

The experiments at Tevatron are performing searches for SUSY particles in several scenarios. Third generation squarks (stop and sbottom) searches are described in this section.

\subsection{Sbottom Searches}

If $\tan \beta$ is large, then there can be a large mass splitting in the scalar bottom sector, yielding a mass to the lightest state $(\tilde{b})$ in the reach of the Tevatron center of mass energy. Assuming R-parity conservation the $\tilde{b}$ decay has to involve the LSP in the final state.

At Tevatron, two different searches for sbottom are performed depending on its production mechanism. Direct $\tilde{b}$ production with the subsequent sbottom decay to a b-quark and the lightest neutralino $\left(\tilde{\chi}^{0}\right)$, leads to the main signature for $\tilde{b}$ detection which includes two $b$ jets and $\not_{T}$. The other one is the $\tilde{b}$ production through gluino ( $\tilde{g}$ ) decays. Under the assumption that mass of the $\tilde{g}$ is smaller than the mass of the $\tilde{q}$, but larger than mass mass of the $\tilde{b}$, the gluino pair production, $p \bar{p} \rightarrow \tilde{g} \tilde{g}$ is one of the dominant SUSY processes. After production the gluino decays to $\tilde{g} \rightarrow b \tilde{b}$ with the subsequent sbottom decay to a b-quark and $\tilde{\chi}^{0}, \tilde{b} \rightarrow b \tilde{\chi}^{0}$.

Although involving more particles and constraints in the SUSY spectrum, this last approach is strongly motivated by the fact that the gluino pair production cross section is large $(\sigma(g \tilde{g}) \sim$ $10 \times \sigma(b \tilde{b}))$ compared to direct sbottom pair production of similar mass.

Since both analysis have $b$ jets in the final state, applying $b$ tagging algorithms are mandatory tools to enhance the sensitivity to the signal and to reduce backgrounds contributions. The $B$ hadrons in jets coming from $b$ quark fragmentation have an average flight path of about a few millimeters, yielding secondary vertices relative to the interaction point (primary vertex). The tagging algorithms are optimized to find these secondary vertices using different approaches in each experiment.

The search for direct $\tilde{b}$ production [3] is performed by the D 0 collaboration using $4 \mathrm{fb}^{-1}$ of data while the search for $\tilde{b}$ production through $\tilde{g}$ decays [ $₫$ ] is performed by the CDF collaboration using $2.5 \mathrm{fb}^{-1}$ of data. In both analyses the results are in good agreement with the SM prediction and no hint of sbottom have been found. They were used to extract exclusion limits for the cross section of 



Figure 1: Exclusion limits at 95\% C.L in the neutralino-sbottom mass plane (left) for direct $\tilde{b}$ production and in the sbottom-gluino mass plane (right) for $\tilde{b}$ production through $\tilde{g}$ decays

the described process. Figure 1 shows the the exclusion limits at 95\% C.L in the neutralino-sbottom mass plane for direct $\tilde{b}$ production and in the sbottom-gluino mass plane for $\tilde{b}$ production through $\tilde{g}$ decays.

\subsection{Stop Searches}

Due to the large mass of the top quark, the mass splitting between the two stop quarks states $\left(\tilde{t}_{1}, \tilde{t}_{2}\right)$ may be large, allowing $\tilde{t}_{1}$ to possibly be the lightest squark, and possible even lighter than the top quark, equation 2.1.

$$
m^{2}\left(\tilde{t}_{1,2}\right)=\frac{1}{2}\left[m^{2}\left(\tilde{t}_{R}\right)+m^{2}\left(\tilde{t}_{L}\right)\right] \mp \frac{1}{2} \sqrt{\left[m^{2}\left(\tilde{t}_{R}\right)-m^{2}\left(\tilde{t}_{L}\right)\right]^{2}+4 m^{2}(t)\left[A_{t}-\mu \tan \beta\right]^{2}}
$$

Three different searches for $\tilde{t}_{1}$ particles are performed at Tevatron depending on their decay mode (i.e. final state).

The first scenario is accessible in the range $m_{\tilde{t}_{1}}<m_{b}+m_{\tilde{\chi}^{+}}$and $m_{\tilde{t}_{1}}<m_{W}+m_{b}+m_{\tilde{\chi}^{0}}$. The dominant $\tilde{t}_{1}$ decay mode in this model is the flavor changing process $\tilde{t}_{1} \rightarrow c \tilde{\chi}^{0}$ which is typically assumed to occur with $100 \%$ branching fraction. The $\tilde{t}_{1} \rightarrow t \tilde{\chi}^{0}$ decay is kinematically forbidden over the $\tilde{t}_{1}$ mass range currently accessible at Tevatron, and the tree level four-body decays $\tilde{t}_{1} \rightarrow$ $b f f^{\prime} \tilde{\chi}^{0}$ can be neglected. In this particular case the experimental signature consists of two c-jets and $\not_{T}$ from the undetected $\tilde{\chi}^{0}[$ [\$] .

In the second scenario we assume that $\operatorname{BR}\left(\tilde{t}_{1} \rightarrow b l \tilde{v}\right)=1$, where $\tilde{v}$ is the scalar neutrino (sneutrino). Among possible stop decays, this final state is one of the most attractive from the experimental point of view; in addition to $a b$ quark, it benefits from the presence of a lepton with high transverse momentum with respect to the beam axis. The sneutrino is either the LSP or decays invisibly: $\tilde{v} \rightarrow v \tilde{\chi}^{0}$ or $v \tilde{G}$ where the lightest neutralino, $\tilde{\chi}^{0}$, or the gravitino, $\tilde{G}$, is the LSP. 


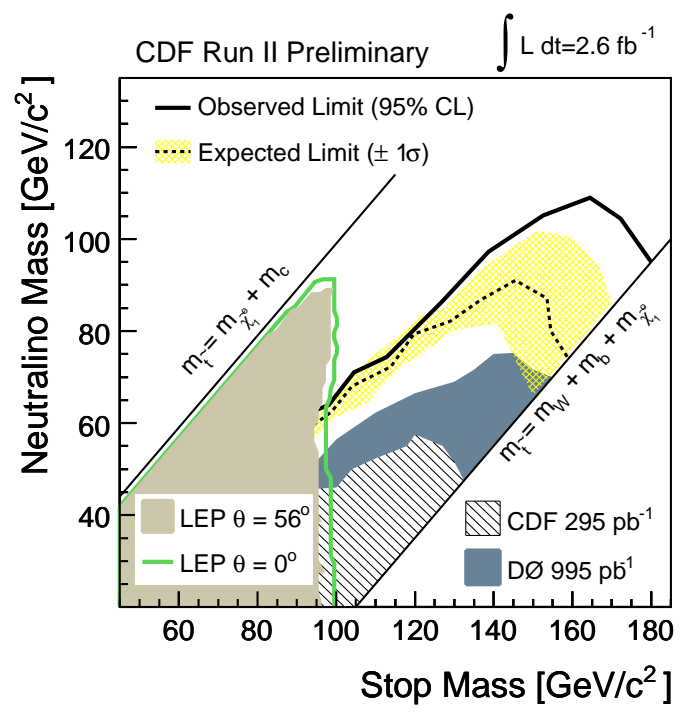

Figure 2: Exclusion limits at $95 \%$ C.L in the neutralino-stop mass plane for stop decaying into charm+neutralino at CDF.

The signal topology consists of two isolated leptons, $\mathbb{E}_{T}$, coming mainly because of undetected sneutrinos, and jets [3, 5].

The third scenario is realized when $\tilde{t}_{1} \rightarrow b \tilde{\chi}^{+} \rightarrow b \tilde{\chi}^{0} l v$ assuming a $100 \%$ branching ratio of the stop squark into a $b$ quark and chargino, and allow for the chargino to decay through a variety of channels to the dilepton mode. These stop events produce signatures similar to those of SM top quark decays, and could potentially be hiding in the top samples of the Tevatron data. A specific analysis was made by the D0 collaboration when the chargino decays into $\tilde{\chi}^{0} W$ assuming one $W$ boson decaying to hadrons and the other one to an electron or muon and a neutrino [6].

No significant deviation from the SM prediction was observed in any of the previous searches. The results were used to extract exclusion limits for the cross section of the described processes. Figure 1 a shows the the exclusion limits at $95 \%$ C.L in the neutralino-stop mass plane for stop decaying into charm+neutralino, the exclusion limits at 95\% C.L in the sneutrino-stop mass plane for stop decaying into $\tilde{b} l \tilde{v}$ at D0 and CDF are shown in fig. 3. The exclusion limits at 95\% C.L in the neutralino-stop mass plane for stop decaying into $b \tilde{\chi}^{0} l v$ is shown in fig. 4 . Figure $\emptyset$ also shows the exclusion limit in term of cross section for stop decaying into $b \tilde{\chi}^{0} l v+$ jets.

\section{Conclusions}

The most recent results on searches for third generation squarks at the Tevatron in events with large missing transverse energy, leptons, and multiple jets in the final state have been presented. No evidence of New Physics has been found yet and stringent exclusion limits have been extracted for the production of sbottom and stop quarks predicted in supersymmetric extension of the SM. With almost $6 \mathrm{fb}^{-1}$ of data already collected, CDF and D0 collaborations could reveal hints of New Physics, or place more severe limits on the SUSY parameter space before the start-up of the LHC. 

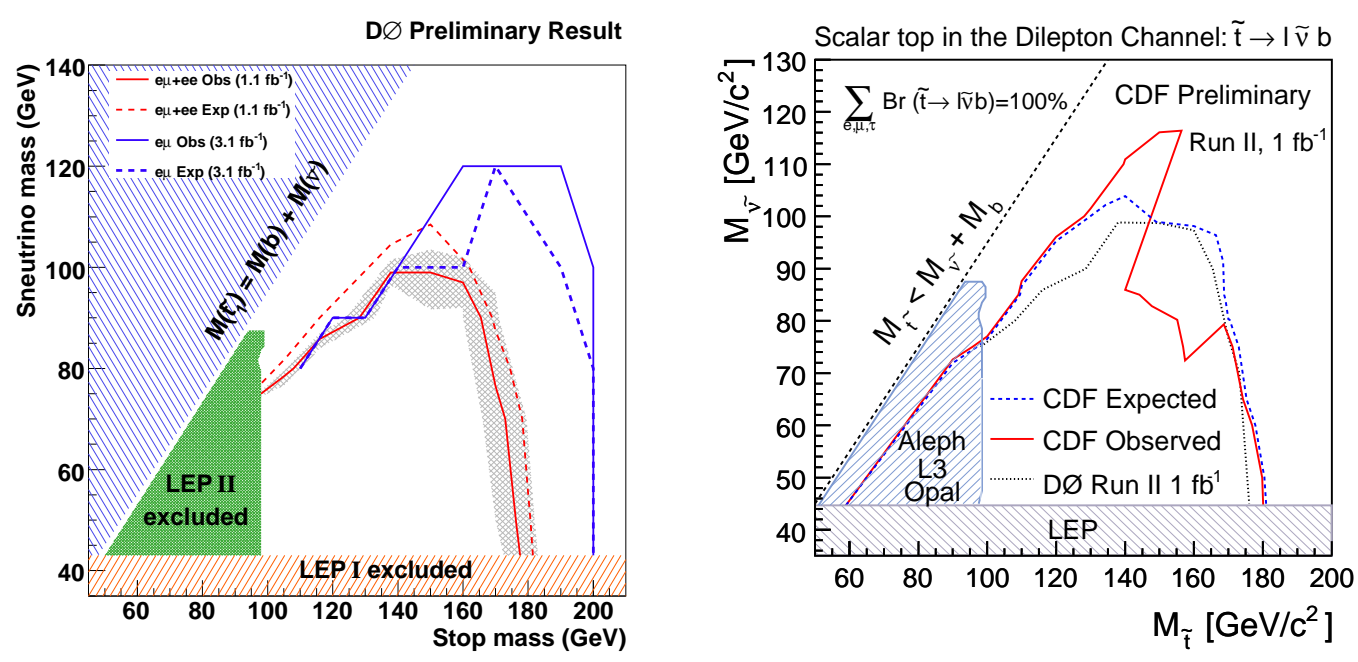

Figure 3: Exclusion limits at 95\% C.L in the sneutrino-stop mass plane for stop decaying into bottom+lepton+sneutrino. Searches performed by D0 (left) and CDF (right) collaborations.
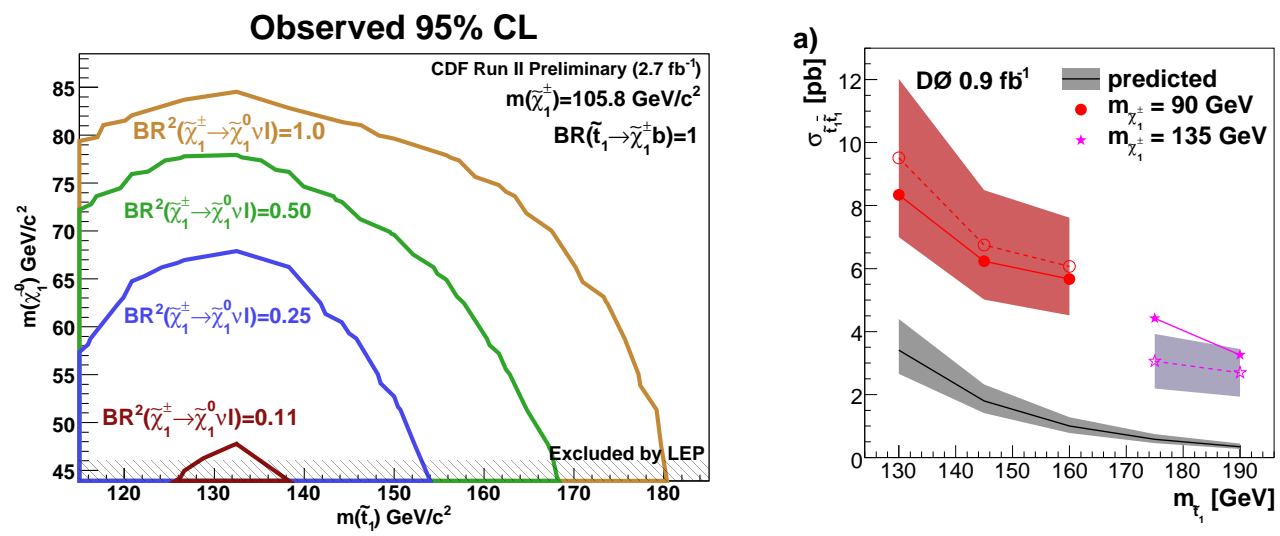

Figure 4: Exclusion limits at 95\% C.L in the neutralino-stop mass plane for the stop decaying into $b \tilde{\chi}^{0} l v$ assuming different branching ratios (left). Cross section limit as a function of the stop mass for stop decaying into $b \tilde{\chi}^{0} l v+$ jets (right).

\section{References}

[1] J. Wess and B. Zumino, Supergauge Transformations in Four-Dimensions, Nucl. Phys B70 (1974).

[2] $\mathrm{R}_{P}=(-1)^{3(B-L)+2 S}$, where $\mathrm{B}$ is the baryon number, $\mathrm{L}$ is the lepton number, and $\mathrm{S}$ is the spin.

[3] http://www-d0.fnal.gov/Run2Physics/WWW/results/np.htm

[4] T. Aaltonen et al. (CDF Collaboration), Search for Gluino-Mediated Bottom Squark Production in $p \bar{p}$ Collisions at $\sqrt{s}=1.96$ TeV, Phys. Rev. Lett. 102 (2009) 221801 [hep-ex/0903.2618].

[5] http://www-cdf.fnal.gov/physics/exotic/exotic.html

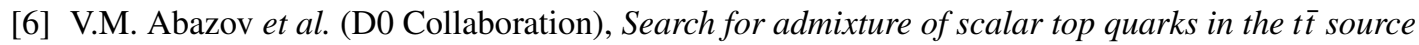
lepton + jets final state at $\sqrt{s}=1.96$ TeV Phys. Lett. B 674 (2009) 4 [hep-ex/ 0901.1063$].$ 